\title{
PRRT: Defining the Paradigm Shift to Achieve Standardization and Individualization
}

\author{
Lisa Bodei ${ }^{1,2}$, Mark Kidd ${ }^{2}$, Richard P. Baum ${ }^{3}$, and Irvin M. Modlin ${ }^{2}$ \\ ${ }^{1}$ Division of Nuclear Medicine, European Institute of Oncology, Milan, Italy; ${ }^{2}$ Department of Gastroenterological Surgery, Yale \\ University School of Medicine, New Haven, Connecticut; and ${ }^{3}$ THERANOSTICS Center for Molecular Radiotherapy and Molecular \\ Imaging, Zentralklinik Bad Berka, Bad Berka, Germany
}

\begin{abstract}
Peptide receptor radionuclide therapy is a treatment for inoperable or metastatic neuroendocrine tumors. A key issue is the need to standardize the treatment and develop randomized controlled trials. Standardization would help define the characteristics of response, including progressionfree survival; provide homogeneous phase II and III studies; delineate the position of peptide receptor radionuclide therapy in the therapeutic algorithm for neuroendocrine tumors; and establish the basis for approval by the regulatory authorities. Standardization of treatments is the starting point to redefine the treatment paradigm from a one-size-fits-all to a personalized treatment. To delineate the treatment paradigm, treatments should be optimized for efficacy and minimization of long-term toxicity, through dosimetry, and adapted to each individual, including relevant patient characteristics. Although differences in therapy outcomes may be explained by the specific absorbed dose (or biologically effective dose), they may also be related to discrete tumor- and patient-specific features. In this respect, a particular area of investigation is the assessment of genetic elements regulating tumor cell proliferation, especially those involved in the response to cytotoxic therapies.
\end{abstract}

Key Words: PRRT; standardization; individualization

J Nucl Med 2014; 55:1753-1756

DOI: 10.2967/jnumed.114.143974

Peptide receptor radionuclide therapy (PRRT) with ${ }^{90} \mathrm{Y}$ - or ${ }^{177} \mathrm{Lu}$-labeled octreotide derivatives is a treatment for inoperable or metastatic, well or moderately differentiated neuroendocrine tumors (NETs) (1). A key issue is the need to standardize the treatment. This is the starting point to define the criteria for personalized therapy.

${ }^{90}$ Y-octreotide was introduced in 1996. Several different clinical protocols arose in Basel, Rotterdam, Bad Berka, and Milan and thereafter throughout Europe, as well as in Asia, Australia, and North America. In 2000, ${ }^{177}$ Lu-octreotate became more widely used because it exhibited greater somatostatin receptor 2 affinity. Subsequently, despite the absence of demonstration of a clear superiority, it has become the radiopeptide of choice given its favorable response rates, lower incidence of toxicity, and synchronous imaging and dosimetry.

Received May 29, 2014; revision accepted Aug. 12, 2014.

For correspondence contact: Lisa Bodei, Division of Nuclear Medicine,

European Institute of Oncology, via Ripamonti 435, Milan 20141, Italy.

E-mail: lisa.bodei@ieo.it

Published online Sep. 25, 2014.

COPYRIGHT (C) 2014 by the Society of Nuclear Medicine and Molecular Imaging, Inc.
${ }^{90}$ Y-octreotide and ${ }^{177} \mathrm{Lu}$-octreotate have been widely investigated in clinical trials worldwide (2). However, $20 \mathrm{y}$ after its introduction, PRRT still uses orphan drugs and includes protocols that differ in selection and treatment criteria. Each protocol represents the research and clinical predispositions of a single local institution. As a consequence, PRRT lacks unequivocal and robust efficacy and toxicity data and has therefore not become a standardized tool in the therapeutic algorithms of NET management (3).

Standardization is a critically necessary step and would define the characteristics of response, including PFS; establish homogeneous phase II and III studies; and delineate the position of PRRT in the therapeutic algorithm for NETs.

Standardization represents the basis for therapeutic optimization, within the framework of maximizing efficacy while minimizing toxicity. To accomplish this goal, a data matrix can be developed by defining patient-specific treatment plans that interface the individual tumor and normal-tissue characteristics with the radiotherapeutic activity, as has been established for external radiotherapy.

\section{CURRENT STATUS}

Unresectable or metastatic well or moderately differentiated gastroenteropancreatic or bronchopulmonary NETs are considered ideal candidates for PRRT (1). In these, a survival impact and objective responses (partial and complete) have been described in up to $30 \%$ of patients, although in the absence of randomized controlled trials (RCTs) this benefit is not quantifiable (Table 1). A comparative literature analysis indicates that survival in ${ }^{177} \mathrm{Lu}-$ octreotate-responsive individuals exceeded that of conventional therapies by 21-42 mo from initiation of therapy (4).

PRRT is generally well tolerated. Acute side effects are usually mild. Permanent effects on the kidneys and bone marrow are generally mild if necessary precautions, such as nephroprotection, are undertaken (2). Acute hematologic toxicity is mild and transient in approximately $90 \%$ of patients. Severe forms tend to recover about 12 mo from PRRT completion (5). Myeloproliferative events are rare (1\%-2\%). Because of their radiosensitivity, the kidneys represent the critical organs, especially with ${ }^{90}$ Y-peptides (Table 2).

PRRT is usually considered by a multidisciplinary tumor board after treatment with somatostatin analogs and, if possible, after cytoreduction with surgery or locally ablative therapies (2). Everolimus and sunitinib are registered for progressive pancreatic NETs and are currently used before PRRT despite limited data. 
TABLE 1

Clinical Results of PRRT in Gastroenteropancreatic NETs

\begin{tabular}{|c|c|c|c|c|c|c|c|c|}
\hline Therapy & Study & $\begin{array}{l}\text { No. of } \\
\text { patients }\end{array}$ & CR & PR & Schedule & $\begin{array}{l}\text { Progression } \\
\text { at baseline }\end{array}$ & $\begin{array}{l}\text { Response } \\
\text { criteria }\end{array}$ & Outcome \\
\hline \multirow[t]{5}{*}{${ }^{90}$ Y-octrotide } & $\begin{array}{l}\text { Waldherr, } \\
2002 \text { (9) }\end{array}$ & 36 & $4 \%$ & $20 \%$ & $\begin{array}{c}7.4 \mathrm{GBq} / \mathrm{m}^{2} \\
\text { in } 4 \text { cycles }\end{array}$ & $100 \%$ & WHO & Not assessed \\
\hline & $\begin{array}{l}\text { Bodei, } \\
2003 \text { (10) }\end{array}$ & 21 & $0 \%$ & $28 \%$ & $\begin{array}{l}2.96-5.55 \\
\text { GBq/cycle } \times 2\end{array}$ & NA & WHO & TTP, $10 \mathrm{mo}$ \\
\hline & $\begin{array}{l}\text { Valkema, } \\
2006 \text { (11) }\end{array}$ & 58 & $0 \%$ & $9 \%$ & $\begin{array}{l}0.93-2.78 \\
\mathrm{~GB} / \mathrm{m}^{2} / \text { cycle }\end{array}$ & $81 \%$ & SWOG & TTP, $29 \mathrm{mo}$ \\
\hline & $\begin{array}{l}\text { Bushnell, } \\
2010 \text { (12) }\end{array}$ & 90 & $0 \%$ & $4 \%$ & $4.4 \mathrm{GBq} /$ cycle $\times 3$ & $100 \%$ & SWOG & PFS, 16 mo \\
\hline & $\begin{array}{l}\text { Imhof, } \\
2011 \text { (14) }\end{array}$ & 821 & $0.2 \%$ & $38 \%$ & $\begin{array}{l}\text { 1-10 cycles } \\
\text { (median, 2), } \\
\text { various activities }\end{array}$ & NA & RECIST & $\begin{array}{l}\text { NA (mean OS, } \\
\quad 4-60 \mathrm{mo} \text { ) }\end{array}$ \\
\hline \multirow[t]{2}{*}{${ }^{177} \mathrm{Lu}$-octreotate } & $\begin{array}{l}\text { Kwekkeboom, } \\
2008 \text { (4) }\end{array}$ & 310 & $2 \%$ & $28 \%$ & $\begin{array}{r}27.8-29.6 \mathrm{GBq} \\
\text { in 3-4 cycles }\end{array}$ & $43 \%$ & SWOG & PFS, 33 mo \\
\hline & $\begin{array}{l}\text { Bodei, } \\
2011 \text { (13) }\end{array}$ & 39 & $3 \%$ & $31 \%$ & $\begin{array}{c}3.7-29.2 \mathrm{GBq} \text { in } \\
4-6 \text { cycles of } \\
3.7-7.4 \mathrm{GBq}\end{array}$ & $76 \%$ & RECIST & TTP, 36 mo \\
\hline
\end{tabular}

$\mathrm{CR}=$ complete response; $\mathrm{NA}=$ not applicable; $\mathrm{PFS}=$ progression-free survival; $\mathrm{PR}=$ partial response; RECIST = Response Evaluation Criteria in Solid Tumors; SWOG = Southwest Oncology Group; TTP = time to progression; WHO = World Health Organization.

Nevertheless, PRRT is accepted by scientific societies as "promising." However, PRRT is not yet considered to have a definitive position in therapeutic algorithms, given the paucity of evidencebased studies. A definitive position requires RCTs in defined disease categories. The absence of RCTs is due mainly to political and economic reasons, in view of the different local approaches, as well as the heretofore absence of support from big pharmaceutical companies.

Presently, the level of recommendation for PRRT is grade 3A in the European Society for Medical Oncology gastroenteropancreatic NET guidelines (3). This level reflects the fact that systematic reviews of published clinical series, prospective studies on small groups, and retrospective analyses on larger datasets are unable to rigorously define specific intervention strategies, such as the appropriate timing for the introduction of PRRT. For example, the shorter progression-free survival noted in a retrospective study, when PRRT was preceded by chemoembolization, was criticized for the large number of variables included in the multivariate analysis and cohort heterogeneity (6), whereas concerns about toxicity in gastroenteropancreatic NETs treated with everolimus after PRRT have been contradicted by a retrospective analysis, but this was underpowered (7). A recent clinical consensus using the Copenhagen criteria concluded that the present body of data was insufficient to establish the role and sequence of PRRT in relation to liver-focused therapies (8).

PRRT also lacks marketing authorization. The results of an ongoing prospective registration $\mathrm{RCT}$ of ${ }^{177} \mathrm{Lu}$-octreotate in smallintestinal NETs are projected for 2016 (netter-1.com). Other RCTs in specific types of NETs are also planned to start. Until then, PRRT must be used according to national legislation regulating experimental radiopharmaceuticals within the context of clinical trials. A delay in registration may relegate PRRT to the lowest position in the therapeutic algorithm, surpassed by pharmaceuticals whose advantage is that they are registered and are easily available.

TABLE 2

Long-Term PRRT Toxicity in Gastroenteropancreatic NETs

\begin{tabular}{|c|c|c|c|c|c|c|}
\hline Therapy & Study & No. of patients & Follow-up (mo) & Renal toxicity & $\operatorname{MDS}(n)$ & Leukemia $(n)$ \\
\hline \multirow[t]{4}{*}{${ }^{90} Y$-octreotide } & Bodei, 2003 (10) & 40 & 19 & $10 \%$ grade 1 & 0 & 0 \\
\hline & Waldherr, 2002 (9) & 39 & 6 & $3 \%$ grade 2 & 0 & 0 \\
\hline & Valkema, 2006 (11) & 58 & 18 & $3 \%$ grade 4 & 1 & 0 \\
\hline & Imhof, 2011 (14) & 1,109 & 23 & $9.2 \%$ grades $3 / 4$ & 1 & 1 \\
\hline \multirow[t]{3}{*}{${ }^{177}$ Lu-octreotate } & Kwekkeboom, 2008 (4) & 504 & 19 & $0.4 \%$ grade 4 & 3 & 0 \\
\hline & Bodei, 2011 (13) & 51 & 29 & $24 \%$ grade 1 & 0 & 0 \\
\hline & Sabet, 2013 and $2014(5,23)$ & 74 & 21 & $1.3 \%$ grades $3 / 4$ & 3 & 0 \\
\hline
\end{tabular}

MDS = myelodysplastic syndrome. 


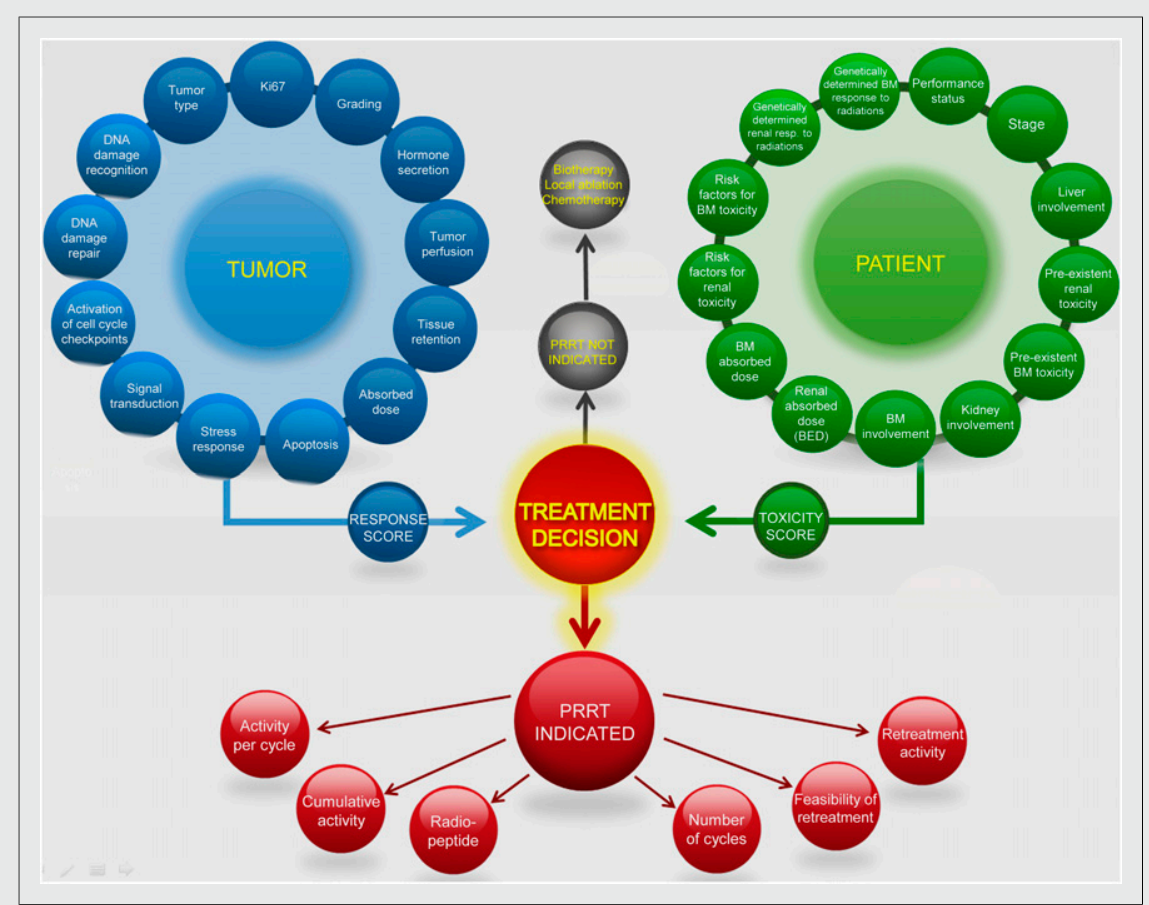

FIGURE 1. Decision analysis for treatment individualization. BED = biologically effective dose; $\mathrm{BM}=$ bone marrow.

\section{TOWARD PERSONALIZED TREATMENT}

Personalized treatment is a strategy to optimize the therapy, minimize the toxicity, and maximize the probability of response. Personalization can be achieved through dosimetry, adapting the administered activity to individuals according to relevant characteristics, but only once the therapy has been well defined and can be provided in a standardized format.

Classically, institutions tend to apply their own PRRT scheme with different inclusion criteria, total and per-cycle administered activities, and numbers and frequencies of cycles $(4,8-14)$. Moreover, PRRT is commonly performed with different but fixed activities. Given the variable absorbed doses delivered to the target tissues, the effects are not entirely predictable. To date, the outcomes of PRRT are best described as "a large probability of efficacy with a low risk of serious toxicity" $(2,8)$. However, this strategy is suboptimal if irreversible toxicity or inefficacy occurs. Toxicity may reduce the tolerance to future therapies targeting the same organs, and inefficient treatments have a significant downside if they induce unnecessary radiation exposure without the desired therapeutic benefit. Adapting the administered activity to performance status, body surface area, and renal and hematologic parameters is a rudimentary form of personalization but fails to consider the variable dosimetric and biologic effects at the target. Dosimetric estimation of the absorbed tumor and normal-organ dose allows optimization. The MIRD and OLINDA/EXM codes used to derive the dose, however, do not represent the organ anatomy of individual patients. Such derivations require complex personalized Monte Carlo simulations.

A relationship between the tumor-absorbed dose and response has been demonstrated for ${ }^{90}$ Y-DOTATOC, indicating that dosimetry-based treatments may improve outcome (15). However, as opposed to external radiotherapy, in which the geometry of the target and the characteristics of irradiation allow quantification, the prediction of the dose-response relationship with radionuclides requires further calibration because of the inability of the absorbed dose to accurately estimate nephrotoxicity and the difficulty in bone marrow and tumor modeling. Other reasons include the variable individual organ volumes, biodistribution, and tumor uptake and the lack of a uniform distribution of radioactivity (16). As a consequence, dosimetry is debated and is not widely accepted. Supporters claim that absorbed doses correlate with clinical outcomes and, ultimately, facilitate the individualization based on the cost-benefit ratio and the clinical need, whether eradication or palliation. Opponents claim dosimetry is timeand resource-consuming, is imprecise, and casts a shadow of complexity and danger on PRRT, since severe toxicity is rare. However, acquisition of knowledge on treatment efficacy and safety is always beneficial for both patients and physicians. In addition, the identification of clinical factors modifying the renal dose threshold to radiopeptides formed the basis for an adaptation of the PRRT schedule (16). When conventional bone marrow and kidney dose thresholds are used, a fixed PRRT schedule results in different absorbed doses. It is thus apparent that individuals may or may not tolerate additional activities or cycles (17).

Dosimetric estimates, however, must be integrated with individual radiobiologic tissue characteristics that have been introduced to better predict the dose-effect relationship. This requirement reflects the observation that differential tumor and normal-tissue proliferation rates represent a major determinant of response. Biologically effective dose and its tissue distribution correlate with specific effects, such as renal toxicity (18). Additional concepts include individual cell radiosensitivity, tumor control probability (depending on the number of clonogenic cells), and normal-tissue control probability (depending on the type of organ architecture). The last of these is of considerable biologic relevance since bone marrow, which exhibits a rapid turnover, responds differently from renal tissue, which has a slow turnover. These models, however, remain theoretic and are not yet clinically applicable. Nonetheless, they emphasize the biologic variability inherent in the irradiated tissues as a major determinant of individual response. The algorithmic assessment of these multiple parameters will allow clinicians to better individualize susceptible or resistant patients.

Although individual differences in therapy outcomes may be explained by the specific absorbed dose (or biologically effective dose), they may also be related to tumor- and patientspecific features such as the biology of the tumor, stage, 
performance status, and risk factors for renal and bone marrow toxicity. Some of these may reflect genetically determined biologic characteristics.

\section{REQUIREMENTS FOR OPTIMIZATION OF INDIVIDUALIZATION}

To redefine the paradigm of treatment, the perspective must be altered from a one-size-fits-all to a personalized treatment. To delineate this personalization, genetic elements regulating tumor cell proliferation, especially those involved in the response to cytotoxic therapies, require additional study. These comprise assessment of tumor cell signal transduction mechanisms and stress response pathways activated by PRRT, evaluation of the expression and radiosensitivity of cell cycle checkpoint proteins, recognition of differences in pathways involved in DNA damage and repair after exposure to PRRT, and what mechanisms regulate apoptotic and premature senescence pathways (19). It is likely that transcriptome-based and sequencing approaches will identify the gene signatures or genetic elements, such as single-nucleotide polymorphisms in the XPF pathway, that confer tumor cell sensitivity to therapy $(20,21)$.

To investigate tumor radiosensitivity, an evaluation of individual tumor tissue is required by analyzing the oxygenation status and developing primary cultures (and potentially cell lines), identifying specific mutations (or single-nucleotide polymorphisms) in known radiosensitive/radioresistance pathways, and evaluating the stress response signaling and proliferative/apoptotic networks. In addition, the radiosensitivity of normal tissues should be evaluated from whole-genome sequencing (single-nucleotide polymorphism-based analyses) of circulating cells or from a targeted sequencing of candidate radiation-sensitive and resistant loci. For a full appreciation of the spectrum of normal and tumor responses in an individual, an evaluation of person- or tumor-specific response to radiation, possibly by a peripheral genomic approach, may also require consideration.

Once the patient-specific sensitivity to radiation has been defined for the tumor, adjacent normal tissue, and the critical at-risk organs, an optimal PRRT schedule can be designed (Fig. 1). Following the current approaches in medical management, a multilevel assessment of these individual variables can be included in a predictive nomogram. This will lead to the design of a specialized PRRT nomogram able to predict efficacy and toxicity and thereby define a risk-benefit assessment strategy for an individual before therapy. In this scenario, the availability of molecular prognostic factors able to identify the tumor and normal-organ response to PRRT will be a necessary step to optimize management (22).

\section{CONCLUSION}

Is this pure fiction or is it a viable strategy? As Julius Caesar noted, "What is possible has been done and what is impossible will be done." As in other treatments, a shift toward personalization according to individual characteristics is an inevitable trend. This strategy will provide the opportunity for nuclear medicine physicians to overcome the current skepticism about the therapeutic benefit of PRRT and allay anxiety about long-term toxicity. However, the road toward personalized PRRT must first pass through the common language of mutual agreement and standardization. The strategy of "we the people" has already been proven.

\section{DISCLOSURE}

No potential conflict of interest relevant to this article was reported.

\section{REFERENCES}

1. Bodei L, Mueller-Brand J, Baum RP, et al. Erratum to: The joint IAEA, EANM, and SNMMI practical guidance on peptide receptor radionuclide therapy (PRRNT) in neuroendocrine tumours. Eur J Nucl Med Mol Imaging. 2014;41: 584.

2. Kwekkeboom DJ, Kam BL, van Essen M, et al. Somatostatin-receptor-based imaging and therapy of gastroenteropancreatic neuroendocrine tumors. Endocr Relat Cancer. 2010;17:R53-R73.

3. Öberg K, Knigge U, Kwekkeboom D, Perren A. Neuroendocrine gastro-enteropancreatic tumors: ESMO clinical practice guidelines for diagnosis, treatment and follow-up. Ann Oncol. 2012;23(suppl 7):vii124-vii130.

4. Kwekkeboom DJ, de Herder WW, Kam BL, et al. Treatment with the radiolabeled somatostatin analog [177 Lu-DOTA 0,Tyr3]octreotate: toxicity, efficacy, and survival. J Clin Oncol. 2008;26:2124-2130.

5. Sabet A, Ezziddin K, Pape UF, et al. Long-term hematotoxicity after peptide receptor radionuclide therapy with ${ }^{177} \mathrm{Lu}$-octreotate. $\mathrm{J} \mathrm{Nucl} \mathrm{Med.} \mathrm{2013;54:1857-1861.}$

6. Campana D, Capurso G, Partelli S, et al. Radiolabelled somatostatin analogue treatment in gastroenteropancreatic neuroendocrine tumours: factors associated with response and suggestions for therapeutic sequence. Eur J Nucl Med Mol Imaging. 2013;40:1197-1205.

7. Kamp K, Gumz B, Feelders RA, et al. Safety and efficacy of everolimus in gastrointestinal and pancreatic neuroendocrine tumors after ${ }^{177} \mathrm{Lu}$-octreotate. Endocr Relat Cancer. 2013;20:825-831.

8. Frilling A, Modlin I, Kidd M, et al. Recommendations for management of patients with neuroendocrine liver metastases. Lancet Oncol. 2014;15:e8-e21.

9. Waldherr C, Pless M, Maecke HR, et al. Tumor response and clinical benefit in neuroendocrine tumors after 7.4 GBq ${ }^{90}$ Y-DOTATOC. J Nucl Med. 2002;43: 610-616.

10. Bodei L, Cremonesi M, Zoboli S, et al. Receptor-mediated radionuclide therapy with ${ }^{90}$ Y-DOTATOC in association with amino acid infusion: a phase I study. Eur J Nucl Med Mol Imaging. 2003;30:207-216.

11. Valkema R, Pauwels S, Kvols LK, et al. Survival and response after peptide receptor radionuclide therapy with $\left[{ }^{90} \mathrm{Y}\right.$-DOTA $\left.{ }^{0}, \mathrm{Tyr}^{3}\right]$ octreotide in patients with advanced gastroenteropancreatic neuroendocrine tumors. Semin Nucl Med. 2006;36:147-156.

12. Bushnell DL Jr, O’Dorisio TM, O’Dorisio MS, et al. ${ }^{90} Y$-edotreotide for metastatic carcinoid refractory to octreotide. J Clin Oncol. 2010;28:1652-1659.

13. Bodei L, Cremonesi M, Grana CM, et al. Peptide receptor radionuclide therapy with ${ }^{177} \mathrm{Lu}-\mathrm{DOTATATE}$ : the IEO phase I-II study. Eur J Nucl Med Mol Imaging. 2011;38:2125-2135.

14. Imhof $A$, Brunner $P$, Marincek $N$, et al. Response, survival, and long-term toxicity after therapy with the radiolabeled somatostatin analogue [ ${ }^{90} \mathrm{Y}$-DOTA]TOC in metastasized neuroendocrine cancers. J Clin Oncol. 2011;29:2416-2423.

15. Strigari L, Konijnenberg M, Chiesa $\mathrm{C}$ et al. The evidence base for the use of internal dosimetry in the clinical practice of molecular radiotherapy. Eur J Nucl Med Mol Imaging. June 11, 2014 [Epub ahead of print].

16. Cremonesi M, Ferrari M, Di Dia A, et al. Recent issues on dosimetry and radiobiology for peptide receptor radionuclide therapy. $Q \mathrm{~J} \mathrm{Nucl} \mathrm{Med} \mathrm{Mol}$ Imaging. 2011;55:155-167.

17. Sandström M, Garske-Roman U, Granberg D, et al. Individualized dosimetry of kidney and bone marrow in patients undergoing ${ }^{177} \mathrm{Lu}$-DOTA-octreotate treatment. J Nucl Med. 2013;54:33-41.

18. Strigari L, Benassi M, Chiesa C, Cremonesi M, Bodei L, D'Andrea M. Dosimetry in nuclear medicine therapy: radiobiology application and results. Q J Nucl Med Mol Imaging. 2011;55:205-221.

19. Parliament MB, Murray D. Single nucleotide polymorphisms of DNA repair genes as predictors of radioresponse. Semin Radiat Oncol. 2010;20:232-240.

20. Hall JS, Iype R, Senra J, et al. Investigation of radiosensitivity gene signatures in cancer cell lines. PLoS ONE. 2014;9:e86329.

21. Vaezi A, Wang X, Buch S, et al. XPF expression correlates with clinical outcome in squamous cell carcinoma of the head and neck. Clin Cancer Res. 2011; 17:5513-5522.

22. Modlin IM, Drozdov I, Kidd M. The identification of gut neuroendocrine tumor disease by multiple synchronous transcript analysis in blood. PLOS ONE. 2013;8:e63364.

23. Sabet A, Ezziddin K, Pape UF, et al. Accurate assessment of long-term nephrotoxicity after peptide receptor radionuclide therapy with ${ }^{177} \mathrm{Lu}$-octreotate. Eur J Nucl Med Mol Imaging. 2014;41:505-510. 